\title{
Outcomes of dental and craniofacial oseeointegrated implantation in head and neck cancer
}

Dr. Phillip Moore, A/Prof. Ian Hewson

St. Vincent's Hospital, Melbourne, Australia

\section{INTRODUCTION}

Treatment of head and neck cancer may result in disfiguring and debilitating anatomical changes. Osseointegrated implants may be used in these patients to facilitate attachment of implant-retained dentures or cosmetic prostheses. In this study, we aimed to report the success rate of both dental and craniofacial osseointegrated implants in head and neck cancer patients. Furthermore, we aimed to define the impact of radiotherapy, smoking, and chemotherapy on the success and failure of these implants.

\section{METHODS}

A retrospective audit of patient records was performed of all patients who underwent placement of osseointegrated implants for dental and cosmetic rehabilitation after treatment of head and neck cancer at St. Vincent's Hospital, Melbourne, Australia. Initial data was collected on patient demographics, surgical details, use of prostheses, and details of post-operative patient follow-up. Implants were placed for the attachment of dental prostheses in oral deficits, and for cosmetic prostheses in patients with nasal, orbital, and auricular deficits. Patients who were treated for head and neck cancer with either ablative surgical treatment or primary radiotherapy treatment were included.

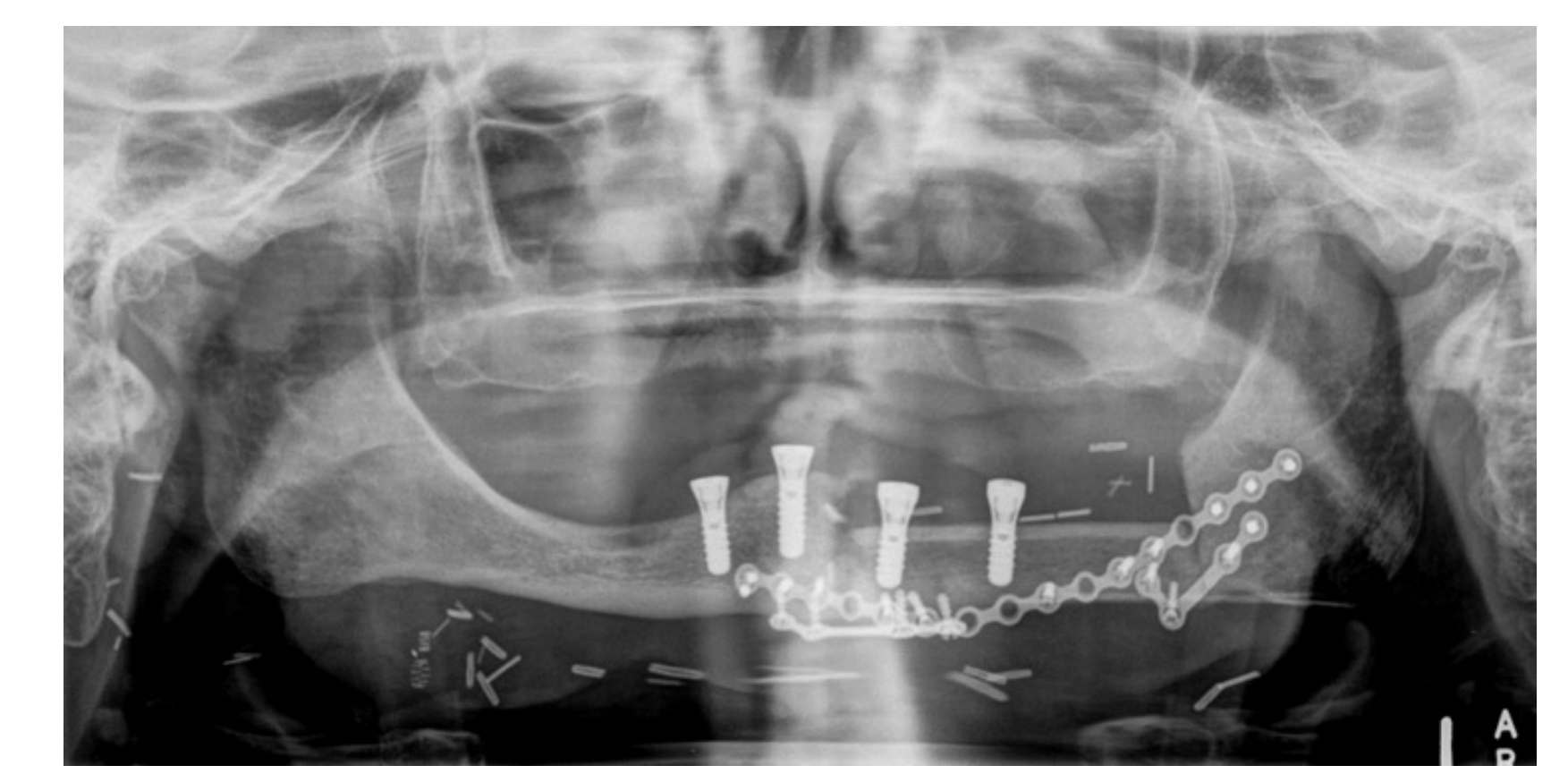

Figure 1: Post-operative orthopantomogram showing two osseointegrated implants in each of native mandible and fibula free-flap.

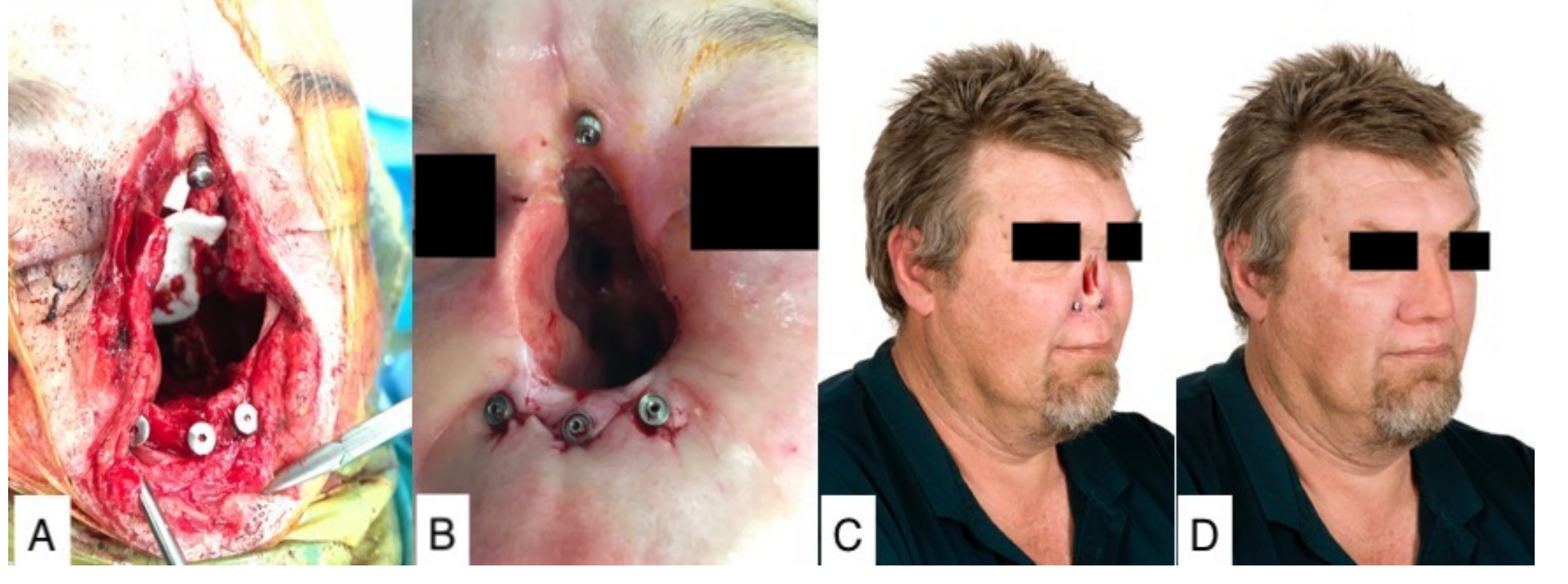

Figure 2: osseointegrated implant-based reconstruction after total rhinectomy. Image A: intraoperative placement of implants in anterior nasal aperture. Image B: After stage 2 - exposure of implants and remodelling of peri-implant soft tissue. Image C and D: After completion of reconstruction, with and without prosthesis.

\section{RESULTS}

160 implants were inserted in 54 patients with oral, nasal, orbital, auricular, or defects. Overall, 136 implants (85\%) were successful after mean follow-up of 25.7 months. The brand of implant used was shown to impart a statistically significant implant survival advantage $(p<0.05)$, and orbital implants had significantly poorer survival than non-orbital implants $(p<0.05)$. There was a statistical insignificant implant survival advantage in both non-smokers ( $p=$ $.108)$ and patients who did not undergo radiotherapy $(p=.090)$. There was no implant survival benefit between implants placed in native bone and bone freeflaps $(p=.489)$.

\section{Gender}

Mean age at time of first implant insertion

Smoking

\begin{tabular}{l} 
Diab \\
Radid \\
Che \\
\hline Tabl \\
\hline Defe \\
\hline Oral \\
Nas \\
Orb
\end{tabular}

Diabetes mellitus

Radiotherapy

Chemotherapy

Table 1: patient demographics

Orbital

Nasal/oral

$\begin{array}{ccc}\text { Implants } & \text { Success } & \text { Radiotherapy } \\ 78(48 \%) & 67(85.9 \%) & 80.8 \% \\ 39(24.4 \%) & 34(87.2 \%) & 82.1 \% \\ 36(22.5 \%) & 28(77.7 \%) & 100 \% \\ 5(3.1 \%) & 5(100 \%) & 100 \% \\ 2(1.3 \%) & 2(100 \%) & 100 \%\end{array}$

41 males $(75.9 \%)$

61.6 years

17 patients $(31.5 \%)$

25 patients $(46.3 \%)$

12 patients $(22.2 \%)$

3 patients $(5.6 \%)$

46 patients $(85.2 \%)$

$62.76 \mathrm{~Gy}$

16 patients $(26.9 \%)$

\section{CONCLUSION}

The use of osseointegrated implants is a reliable method of cosmetic and dental rehabilitation in head and neck cancer patients. Radiotherapy and smoking may increase the risk of implant loss, but overall implant survival rates still remain at $85 \%$. Further research is required to illuminate the role of cigarette smoking in osseointegrated implant failure given the high rates of tobacco use in this patient population.

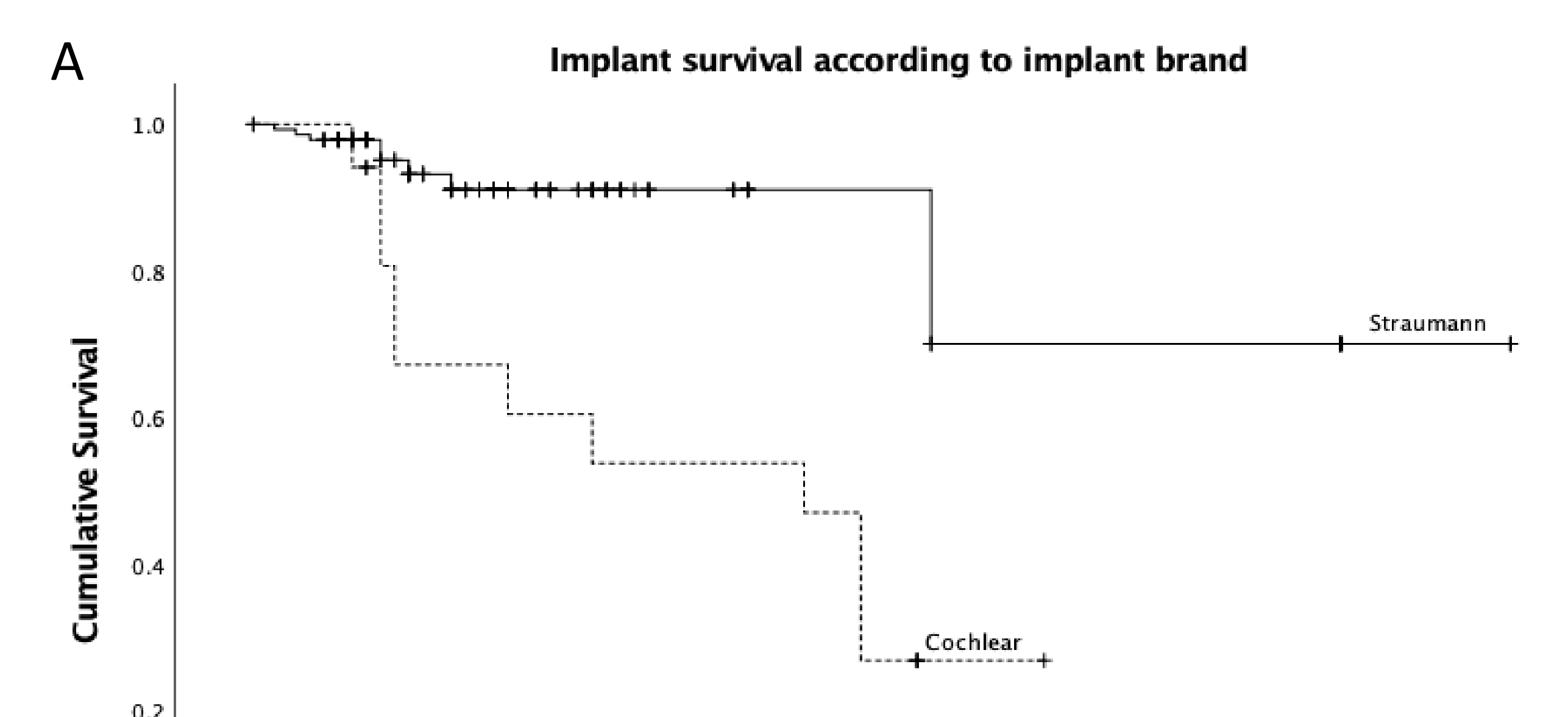

$p<0.05$

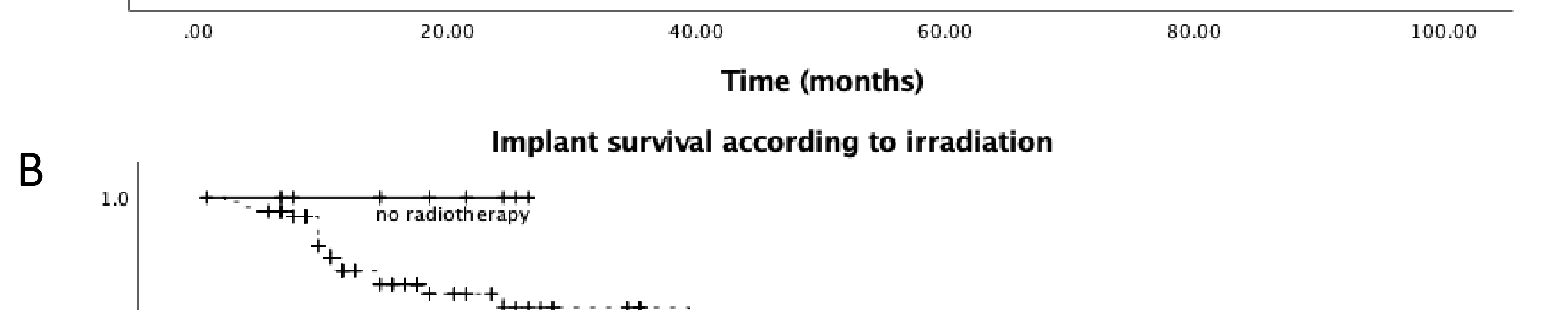

丞 0.6

$p=0.09$
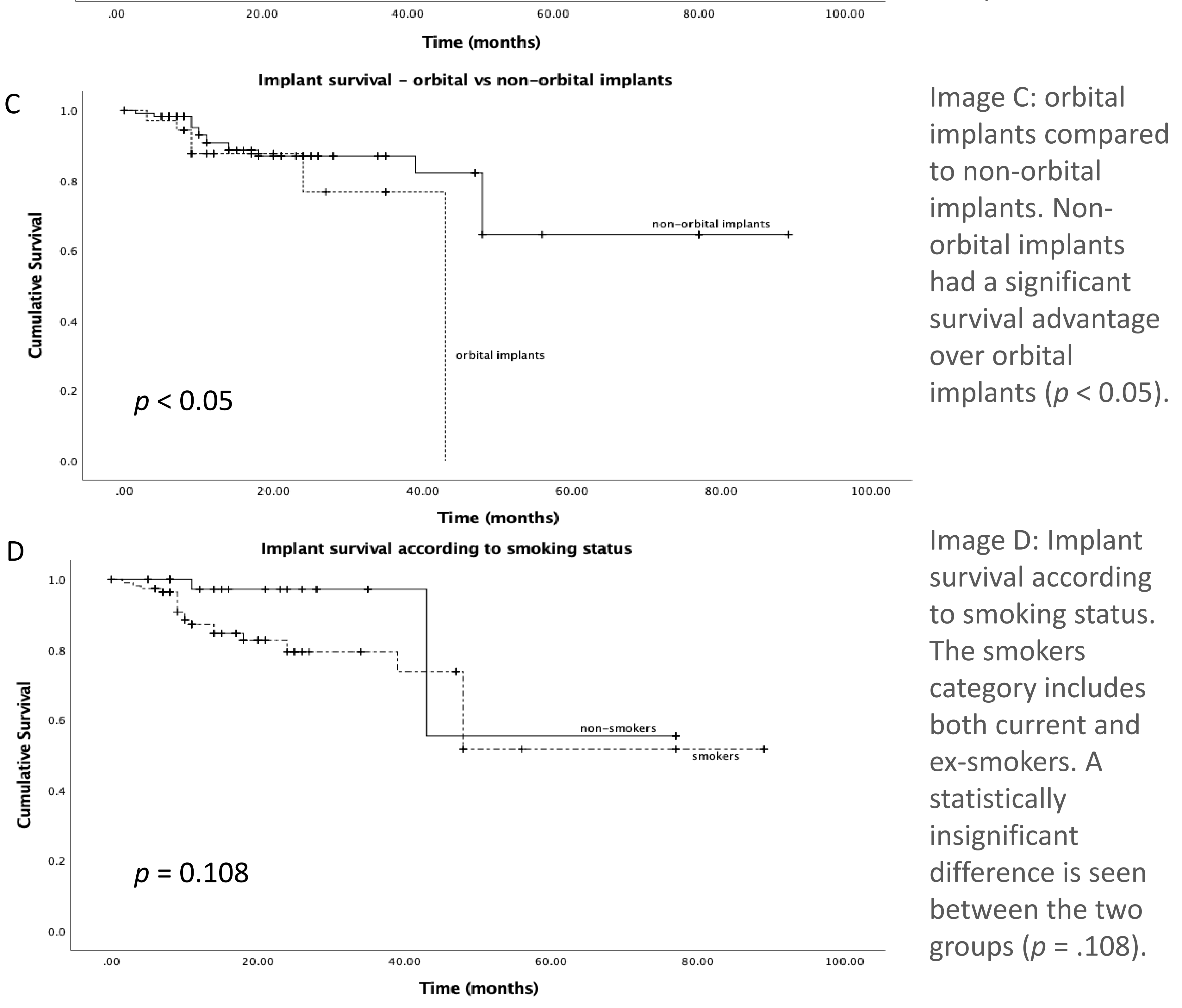

mage D: Implant survival according to smoking status. The smokers category includes both current and ex-smokers. A statistically insignificant difference is seen between the two groups $(p=.108)$

\section{DISCUSSION}

The success rates of oral, nasal, and orbital implants separately are similar to those previously described in comparable studies. It should be noted that $80.8 \%$ of oral implants, $82.1 \%$ of nasal implants, and $100 \%$ of orbital implants were placed in irradiated bone. These percentages are higher than those described in any of the other aforementioned studies. 2, 10-24

The inherent bias of retrospective data collection is the most obvious limitation of this study. Several patients were excluded due to incomplete data sets which may have been avoided if patients had been prospectively recruited monitored, and actively followed-up after the placement of implants. The observed difference between brands of osseointegrated implants must be read with caution given the disparity in experience and volume the operating surgeon had with each brand.

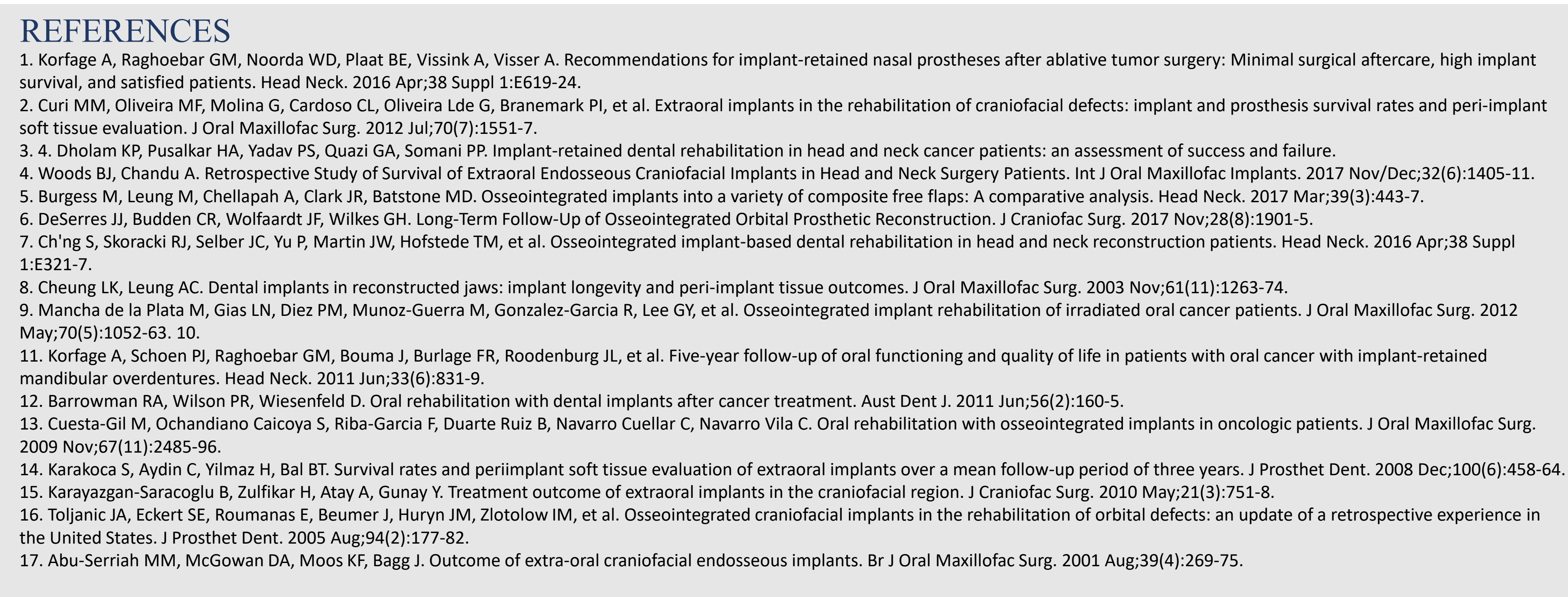

